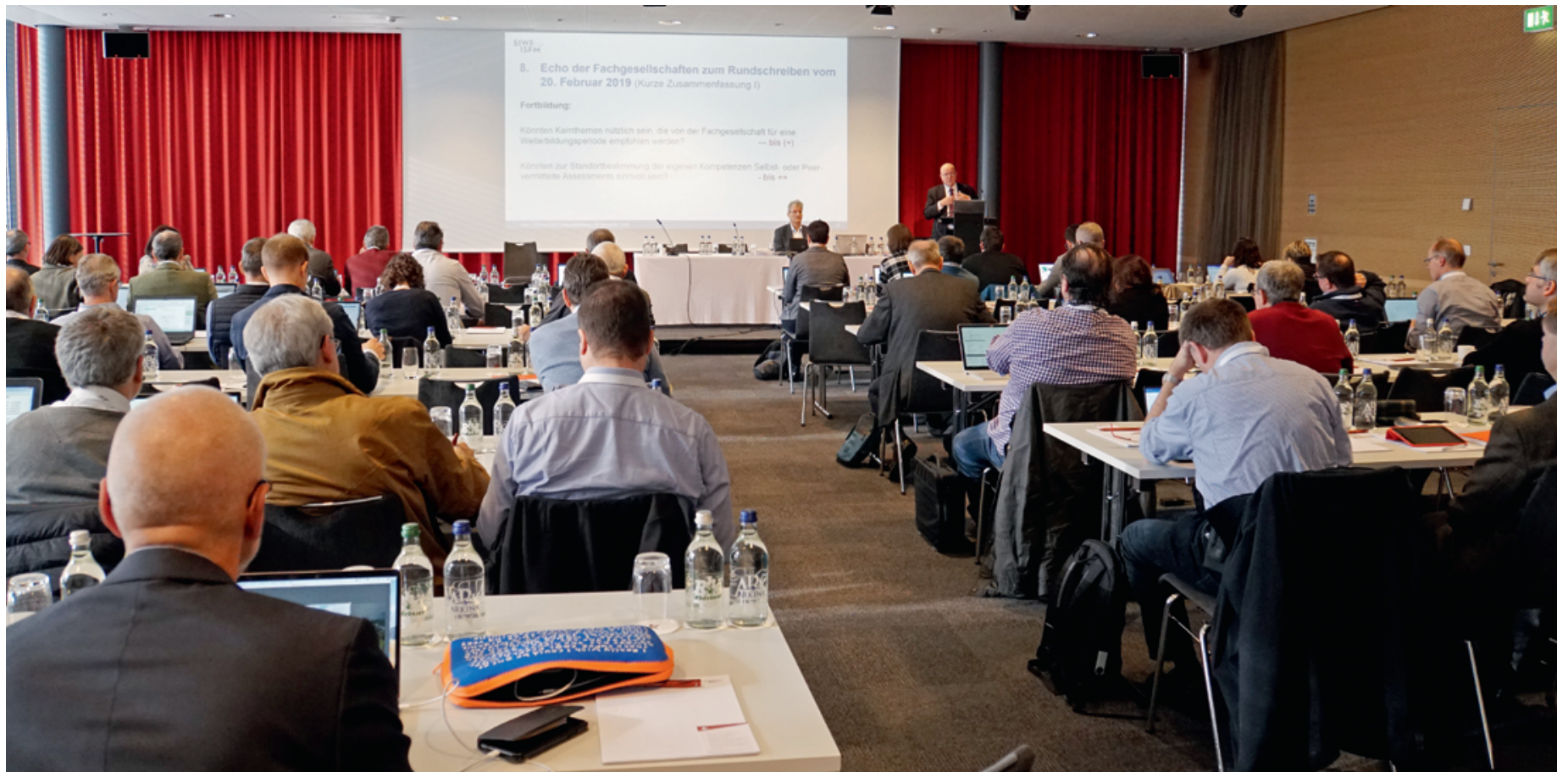

L'assemblée plénière de I'ISFM: une journée intense pour développer la qualité de la formation médicale postgraduée et continue. (fh)

\title{
10 ans de qualité dans la formation
}

Fabienne Hohl

Rédactrice en relations publiques

Dix ans après sa création, l'Institut suisse pour la formation médicale postgraduée et continue (ISFM) poursuit son engagement dans un esprit de clairvoyance et de dialogue, comme en témoigne l'assemblée plénière qui a su allier ordre du jour chargé et discussion avec des invités passionnants. Les délégués ont par ailleurs décidé de poursuivre le développement de la plate-forme de formation continue dans le but de renforcer sa qualité.

«Dans un souci de protection de l'environnement, aucun feu d'artifice ne célébrera les dix ans», a regretté Werner Bauer, président de l'Institut suisse pour la formation médicale postgraduée et continue (ISFM), dans son allocution d'ouverture. Un feu d'artifice aurait pourtant été tout à fait approprié. Après dix ans à la tête de l'ISFM, Werner Bauer passera le flambeau en mai prochain sans avoir rien perdu de la flamme qui l'anime pour la formation médicale. «Dès le début, ce fut un plaisir de travailler avec des gens hautement compétents et engagés aussi bien au sein qu'en dehors de l'ISFM", a-t-il déclaré. Pour lui, développer la formation médicale conjointement avec tous les partenaires a toujours été une motivation fantastique.

\section{Flash d'information: un échange fructueux}

Werner Bauer passe en revue les activités actuelles de l'ISFM et commence par quelques résultats du module destiné aux responsables des établissements de formation postgraduée, qui complète l'enquête que l'ISFM réalise chaque année auprès des médecins-assistants. Cette année, ces questions portaient sur la structure des cursus de formation proposés par une multitude d'établissements de formation postgraduée, seuls ou en collaboration avec d'autres. De ce fait, les réponses obtenues ont été à la hauteur de la diversité qu'ils représentent. Ce qui frappe, c'est la tendance avec laquelle les disciplines populaires, comme la pédiatrie, 


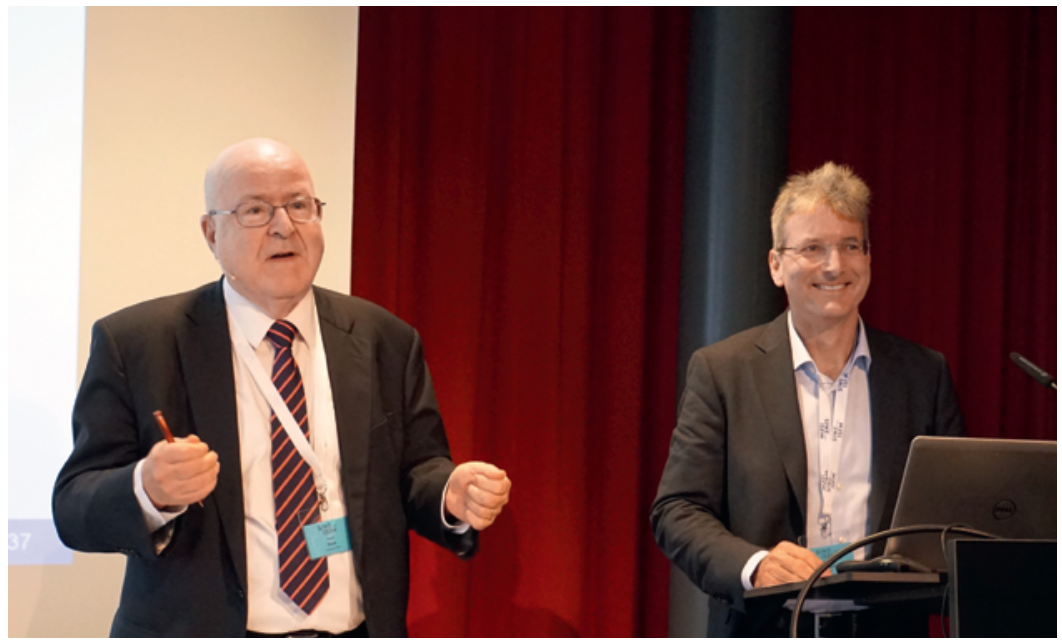

Werner Bauer et Christoph Hänggeli: une excellente équipe qui entretient le contact avec les délégués. (fh)

font état d'un intérêt marqué pour des cursus structurés, contrairement aux disciplines qui rencontrent des problèmes de relève, comme par exemple la psychiatrie.

\section{Manifestations établies}

Werner Bauer a ensuite évoqué trois manifestations de l'ISFM qui jalonnent désormais le calendrier annuel de la formation médicale. Les ateliers Teach the Teachers, que l'ISFM organise en collaboration avec le prestigieux Royal College of Physicians of London, permettent d'approfondir les compétences de leadership. La demande est telle que ces cours seront également proposés en allemand et en français dès 2020. Pour ce faire, le Royal College forme actuellement des responsables de cours pour la Suisse. Toute personne intéres-

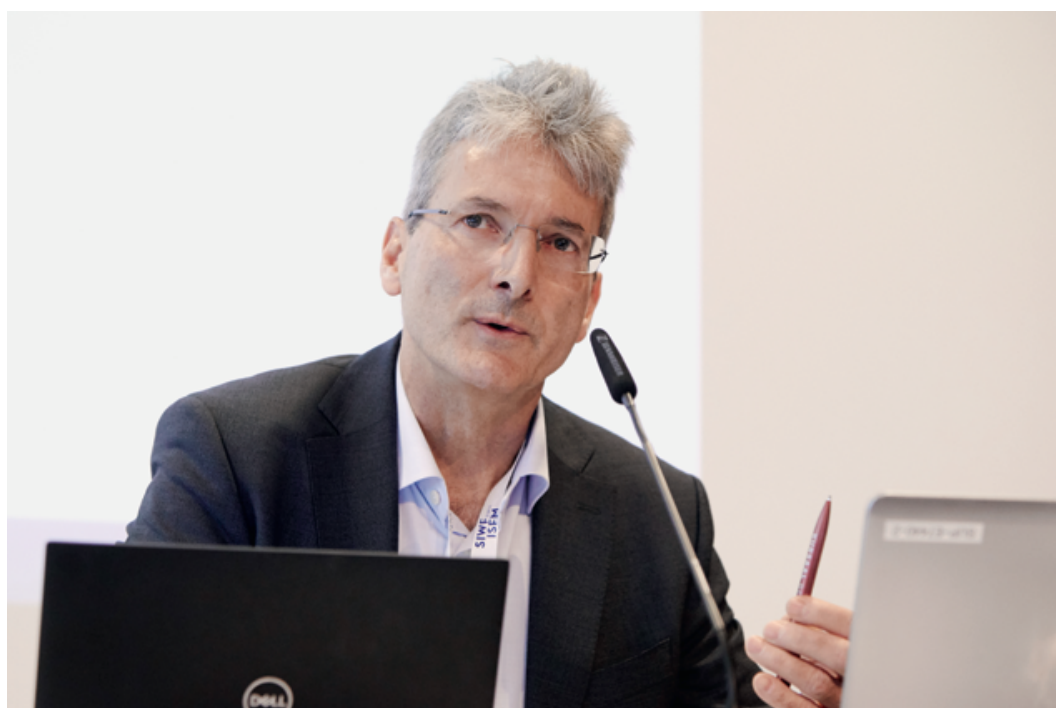

Christoph Hänggeli, directeur de I'ISFM, à propos du budget et des questions juridiques actuelles dans le domaine de la formation postgraduée. (yk) sée par le Medical Teaching peut s'informer auprès de l'ISFM.

Chaque année, l'ISFM convie au symposium MedEd en vue de réfléchir à la formation médicale, et à son évolution, avec le concours d'experts suisses et étrangers. En 2019, le symposium a attiré plus de 200 participants à Berne [1]. Werner Bauer a invité les délégués à proposer des thèmes pour la prochaine édition du 23 septembre 2020 ou à tenir un exposé.

Enfin, l'ISFM organisera cette année la première rencontre dédiée aux équipes de visite. Tous les ans, ce sont plus de 300 établissements de formation postgraduée qui sont reconnus ou classés dans une autre catégorie. Le président a tenu à saluer l'«excellent travail de ces équipes» et rappelé que "grâce à elles, nous garantissons et améliorons de manière patente la qualité de la formation". Avec cette rencontre, l'ISFM souhaite leur offrir un cadre dépassant celui du contrôle des standards et des check-lists. Ici aussi, Werner Bauer a lancé un appel à idées.

\section{LPMéd: la gestion des admissions pose des questions linguistiques}

Christoph Hänggeli a informé les délégués des derniers développements concernant la loi sur les professions médicales (LPMéd). Les exigences linguistiques définies dans la révision de la LPMéd en vigueur depuis janvier 2018 continuent de préoccuper l'ISFM. En plus de la LPMéd, la loi sur l'assurance-maladie exige désormais également, dans le cadre de la gestion des admissions, un examen linguistique de niveau $\mathrm{C} 1$ passé en Suisse. Les détenteurs d'une maturité gymnasiale suisse seront exemptés de l'examen linguistique, mais seulement pour la langue dans laquelle ils ont passé la maturité. Une motion demandant la reconnaissance de toutes les langues inscrites dans la maturité suisse comme preuve des connaissances linguistiques requises est toujours en souffrance.

En 2019, l'ISFM a remporté un succès important au Tribunal fédéral (TF). Il y a deux ans, le Tribunal administratif fédéral avait critiqué le fait que la FMH assume, en tant qu'association de droit privé, des tâches publiques; cela ayant pour conséquence que tous les titres de droit privé (notamment les attestations de formation complémentaire et les formations approfondies) devraient être traités au même titre que les titres fédéraux de spécialiste. «En d'autres termes, toutes les décisions, aussi insignifiantes soient-elles, auraient pu faire l'objet d'un recours devant le Tribunal fédéral», a expliqué Christoph Hänggeli. «Une charge administrative énorme qui exigerait des ressources juridiques bien plus importantes qu'actuellement.» L'ISFM a toujours plaidé que seuls les 


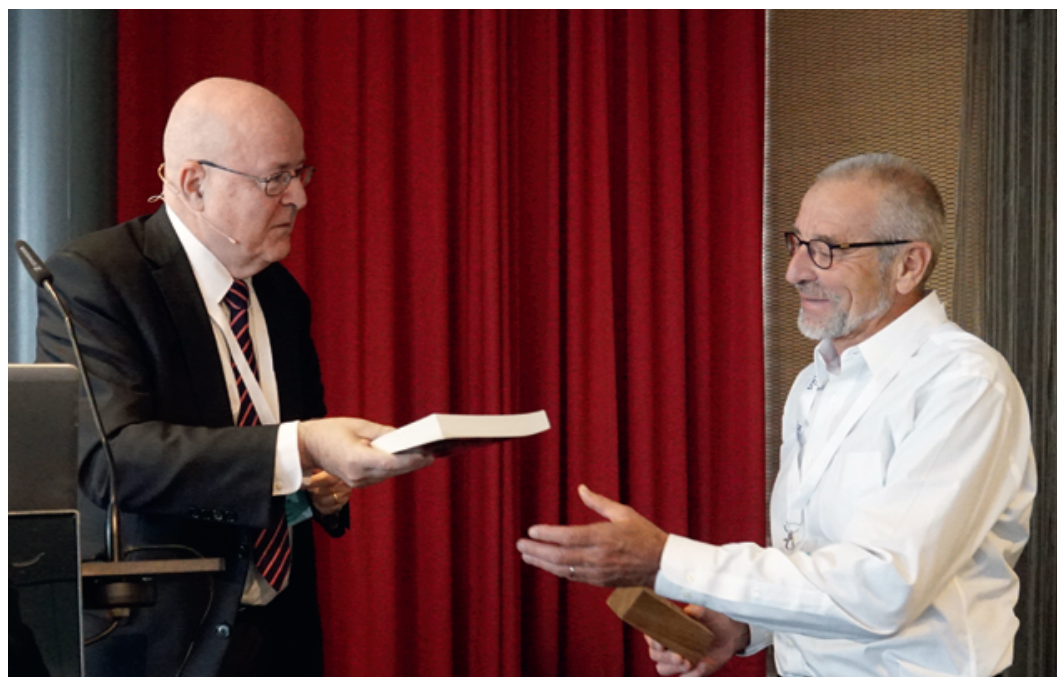

Werner Bauer prend congé de Hans Hoppeler, président démissionnaire de la Section formation postgrade de la Commission des professions médicales. (fh)

45 titres fédéraux de spécialiste étaient soumis à la LPMéd et donc au droit public. Le TF était du même avis et lui a donné raison.

\section{Comptes et budget: une consolidation en vue}

Christoph Hänggeli a ensuite présenté les chiffres de l'ISFM. Les comptes annuels 2018 avaient été clôturés sur une perte inférieure aux prévisions budgétaires. Le budget 2019 prévoit un bilan équilibré. Pour le budget 2020, l'ISFM s'attend à des recettes plus élevées provenant des titres octroyés. Le nombre record de recon-

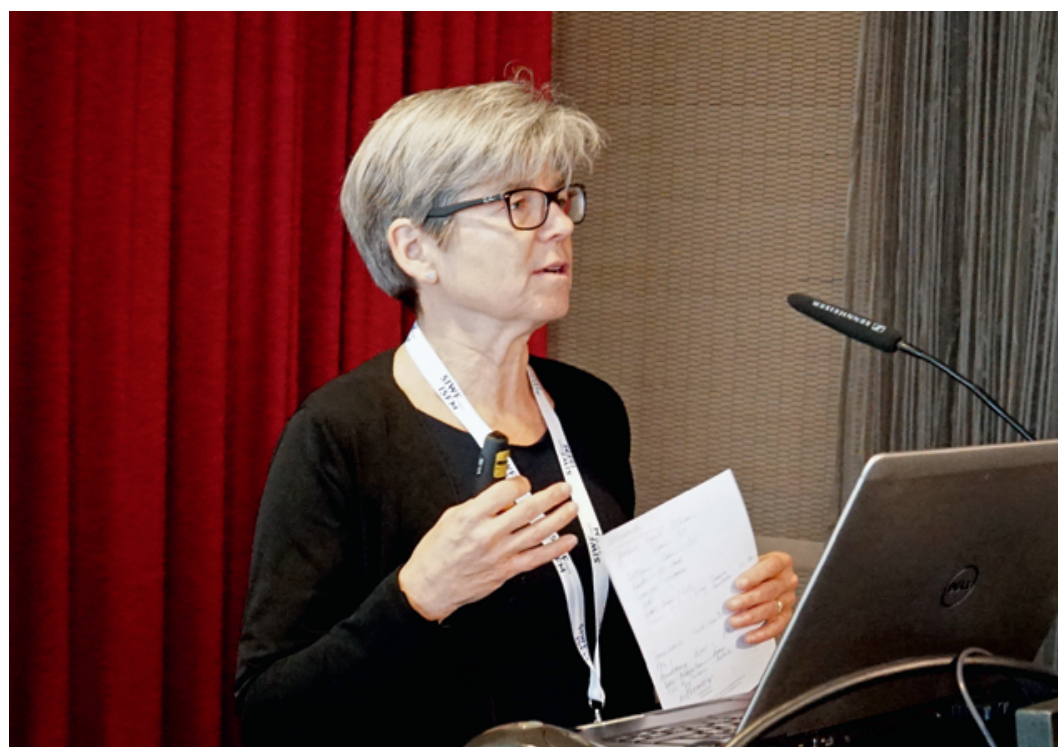

Regula Schmid, ancienne vice-présidente, rend compte de l'introduction des Entrustable Professional Activities (EPA) dans la formation postgraduée. (fh) naissances de diplômes de médecin étrangers et l'augmentation du nombre de places d'étude en Suisse laissent présager une augmentation du nombre de titres de spécialiste. Il faut cependant s'attendre aussi à une augmentation des frais de personnel en raison des nombreuses nouvelles tâches prévues. Le budget prévoit des bénéfices (modérés) à partir de 2020. L'ISFM compte notamment sur des recettes supplémentaires provenant des taxes de certification pour les établissements de formation postgraduée.

\section{Une stratégie vécue}

La stratégie permet de vérifier à intervalles réguliers les objectifs et sous-objectifs de l'ISFM. Les trois objectifs principaux sont le renforcement de la position d'institution nationale de premier plan pour la formation postgraduée et continue des médecins, l'anticipation des évolutions et la conception d'offres de formation tournées vers l'avenir. D’après Werner Bauer, le sous-objectif «Enseignement des objectifs de formation généraux" requiert des mesures [2]. Les médecins-assistants soulèvent régulièrement des déficits à ce sujet lors de l'enquête annuelle de l'EPFZ et de l'ISFM [3]. Le sous-objectif «Élaboration de concepts de formation postgraduée par tous les établissements de formation" n'a pas non plus été atteint pour l'instant. "Ces concepts sont pourtant essentiels, notamment en cas de changements, par exemple pour de nouvelles formes d'organisation", a souligné le président de l'ISFM. Par ailleurs, l'ISFM veut renforcer sa position de centre de compétences leader et indépendant de la formation médicale postgraduée et continue. C'est dans cette perspective qu'il a discuté en novembre 2019 des avantages et inconvénients d'une personnalité juridique propre avec le Comité central de la FMH.

\section{Adieux à Hans Hoppeler}

L'assemblée plénière 2019 n'a pas fait exception à la tradition. Il a fallu prendre congé de Hans Hoppeler, une personnalité qui s'est beaucoup engagée pour la formation médicale. Ce professeur émérite d'anatomie a été longtemps actif comme formateur et président de la Section formation postgrade de la Commission des professions médicales. «Bien que représentant l'autorité, a déclaré non sans humour Werner Bauer en ouverture de son éloge, Hans Hoppeler a notamment largement contribué à l'accréditation de l'ISFM comme centre de compétences pour la formation médicale.» Werner Bauer l'a remercié pour son ouverture d'esprit, ses rapports et ses recommandations innombrables et lui a remis un petit cadeau d'adieu. 


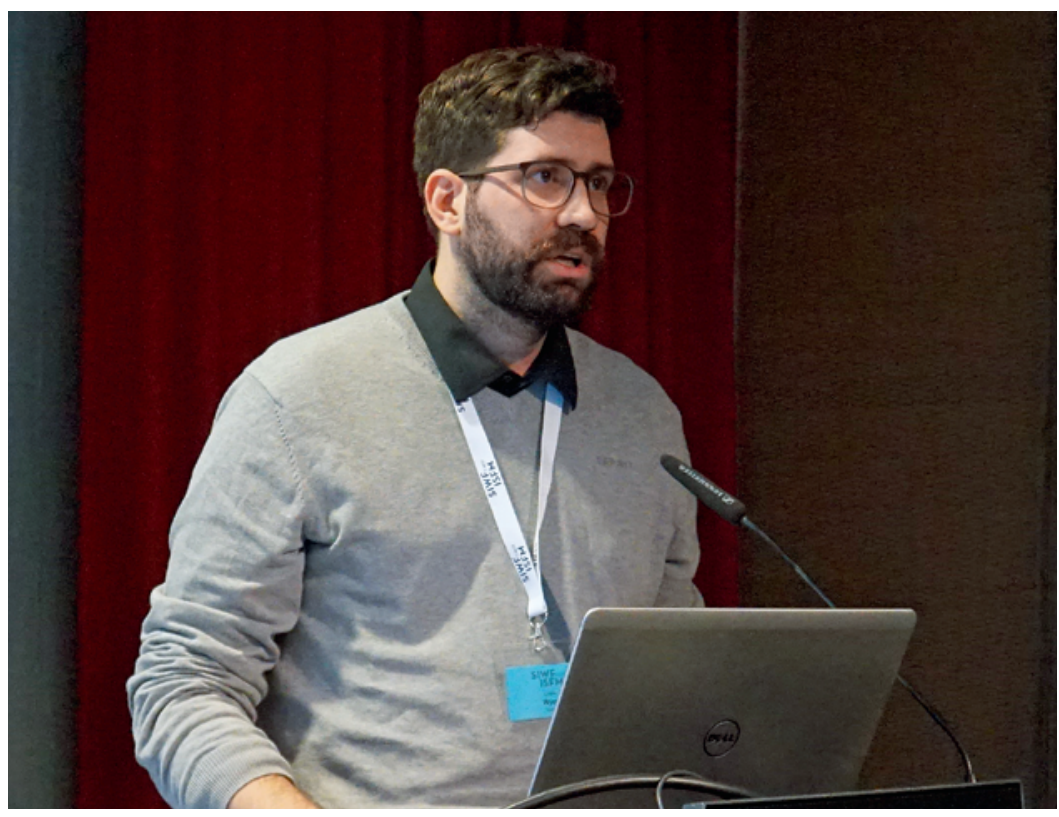

Lukas Wyss informe du bon avancement du logbook électronique. (fh)

\section{EPA sur la bonne voie}

L'ancienne vice-présidente de l'ISFM, Regula Schmid, est chargée de l'introduction des Entrustable Professional Activities (EPA) dans la formation médicale postgraduée [4]. Elle a présenté la prise de position et la nouvelle directive de l'ISFM. Dans de nombreux pays, les EPA sont déjà la norme et se fondent sur le transfert graduel des tâches médicales. En Suisse, les EPA ont du retard, qu'il faut rattraper même si elles font déjà partie de la formation médicale. Le taux élevé de 37 réponses au sondage EPA réalisé auprès de 45 sociétés de discipline a montré "que cette forme moderne de transmission du savoir a trouvé sa place parmi le corps

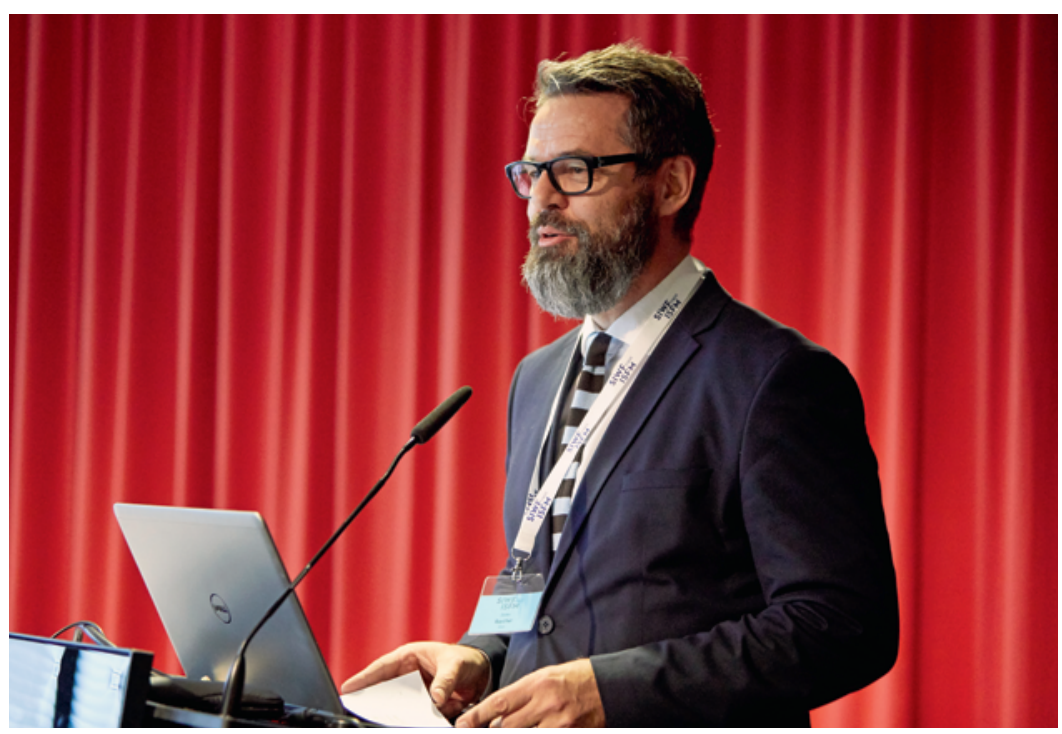

Stefan Spycher, vice-directeur de I'OFSP, apprécie la compétence du travail de I'ISFM. (yk) médical». Les nombreuses questions et suggestions des délégués et les demandes en vue d'un soutien par l'ISFM pour la mise en œuvre des EPA ont souligné le vif intérêt suscité par cette nouvelle approche. La décision de les intégrer dans les programmes de formation postgraduée appartient aux sociétés de discipline. Regula Schmid leur a demandé d'informer l'ISFM au plus tard jusqu'à l'été 2021 de leur position et des activités concernant les EPA. Le groupe de travail EPA de l'ISFM leur apporte son aide dans ce processus, par exemple dans l'atelier EPA du 9 janvier 2020.

\section{Louanges pour le logbook électronique}

L'enquête annuelle auprès des médecins-assistants l'a confirmé: le logbook électronique a définitivement surmonté ses maladies de jeunesse. Plus de la moitié des médecins-assistants se déclarent satisfaits ou très satisfaits. L'informaticien et responsable de projet Lukas Wyss a informé les délégués des progrès réalisés par cet outil essentiel pour l'ISFM. Les bases techniques pour un catalogue des objectifs de formation uniforme selon la nomenclature internationale SNOMED ont été établies et les sociétés de discipline seront invitées en temps voulu à harmoniser leurs objectifs de formation. Cela ne nécessitera pas de modification des programmes de formation ni de la terminologie spécifique: que l'on parle de mastectomie totale ou d'ablation totale du sein, la définition dans SNOMED sera la même pour les deux termes techniques. Lukas Wyss a salué les EPA (voir ci-dessus) car, du point de vue technique, elles complètent les objectifs de formation quantitatifs saisis avec SNOMED par des objectifs qualitatifs.

\section{Message d'anniversaire et pincée de magie}

En guise d'ouverture de l'après-midi, les participants ont eu droit à deux prestations de haut vol. Dans son message d'anniversaire, Stefan Spycher, vice-directeur de l'Office fédéral de la santé publique (OFSP), a reconnu l'excellent travail de l'ISFM et loué la récente accréditation, sans oublier l'échange fructueux dans le cadre de la plate-forme "Avenir de la formation médicale». Quant au deuxième invité, Werner Bauer l'a introduit en tant qu'orateur d'un atelier particulier consacré au leadership. Le magicien mentaliste Pat Perry a effectivement présenté de façon très divertissante avec quelle facilité l'être humain peut être guidé par la présence, la focalisation, l'estime et l'authenticité.

L'assemblée s'est ensuite penchée sur des questions urgentes concernant la formation continue. En effet, ce qui était apparu en 2018 dans le cadre de la plate-forme 


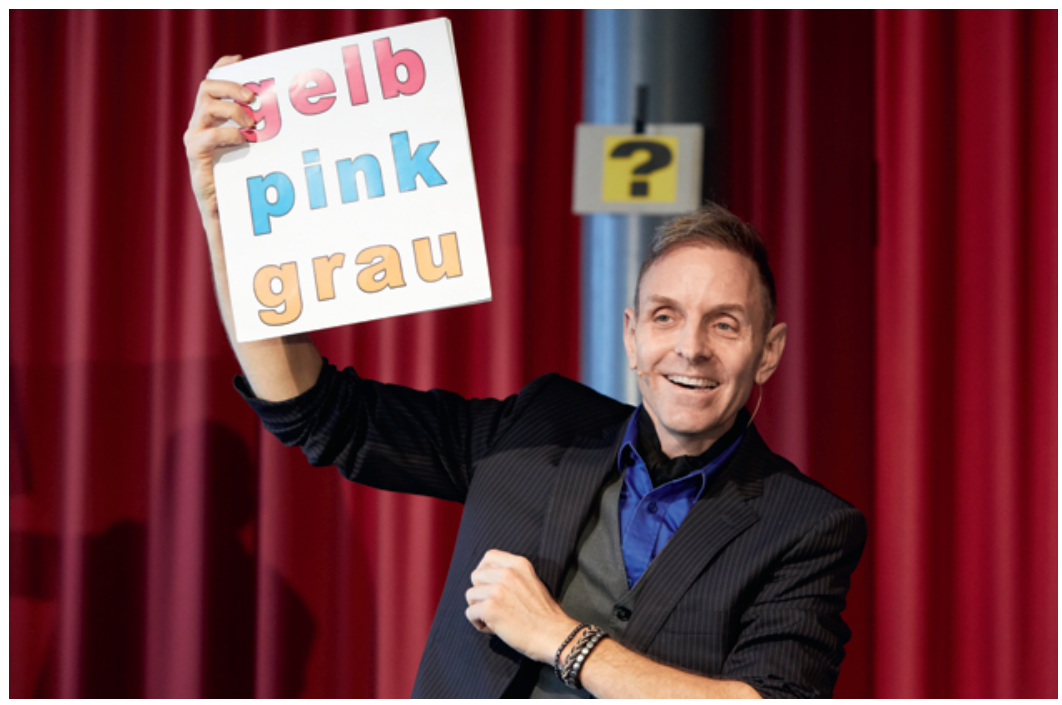

Lors de I'anniversaire de I'ISFM, le magicien mentaliste Pat Perry surprend avec son interprétation des qualités de leadership telles que la présence et la focalisation. (yk)

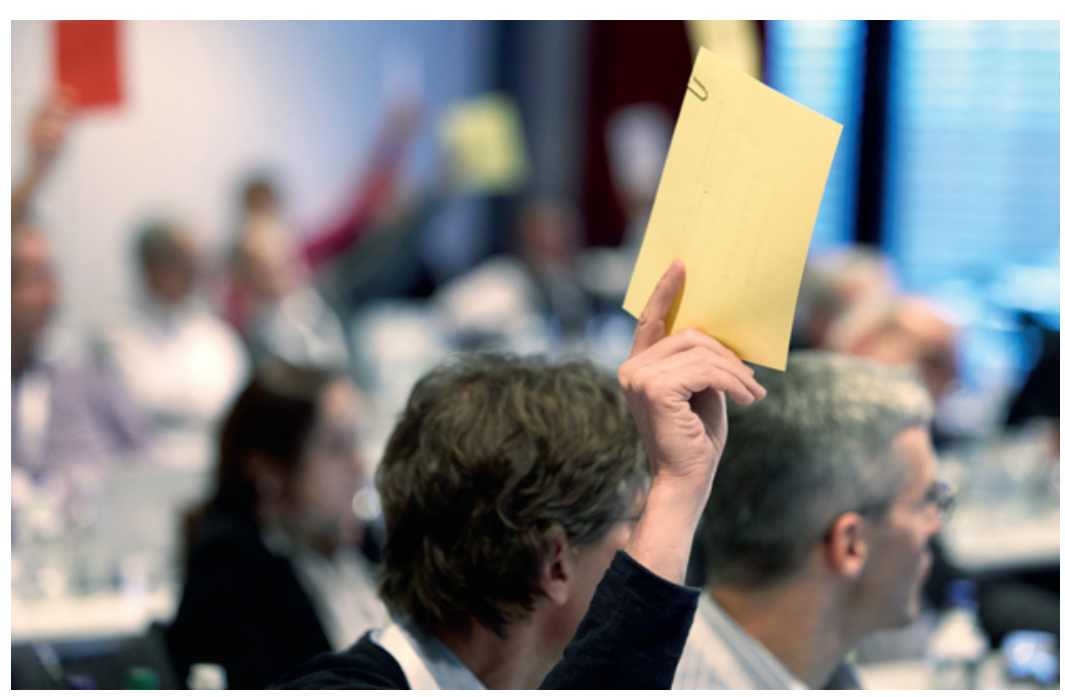

L'assemblée plénière approuve à l'unanimité la précision apportée à la Réglementation pour la formation continue. (yk)

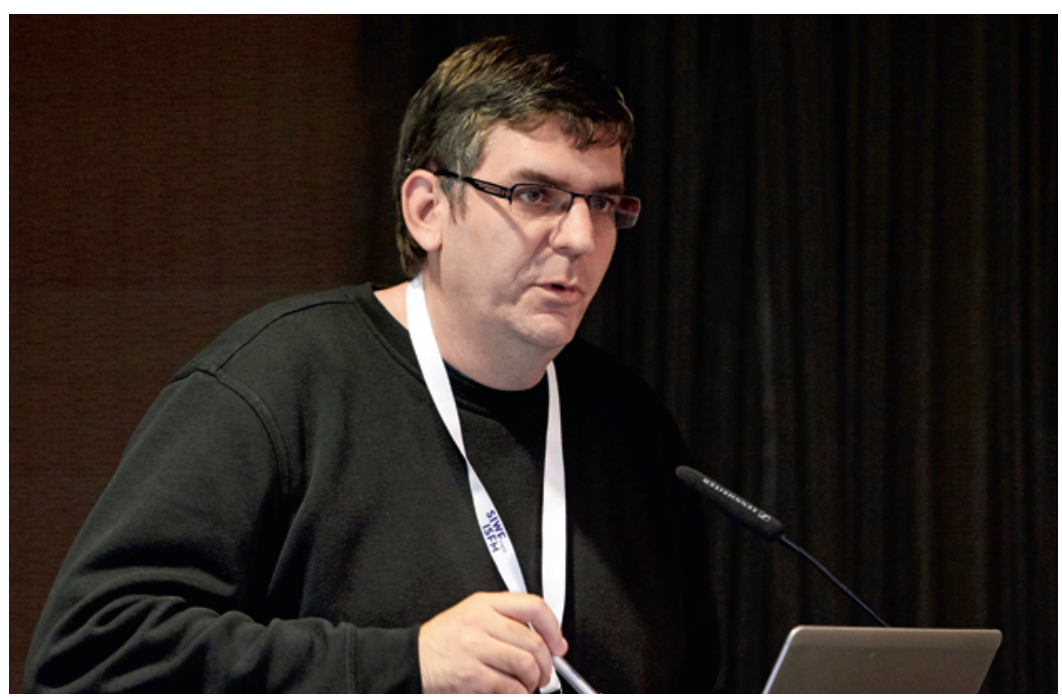

Johnny Graber explique comment la plate-forme de formation continue sera modifiée. (yk)
"Avenir de la formation médicale» s'est transformé en mandat concret pour l'ISFM en l'espace d'une année: l'OFSP et la Conférence suisse des directrices et directeurs cantonaux de la santé ont chargé l'ISFM d'établir un rapport sur la qualité de la formation médicale continue. Le président de l'ISFM, Werner Bauer, s'est clairement prononcé en faveur de l'obligation de formation continue en vigueur fondée sur la responsabilité individuelle, mais a rappelé qu'il ne suffisait pas de collectionner les attestations dans un carton à chaussures. "Sinon, une réglementation imposée de l'extérieur viendra.»

Afin d'assumer au mieux sa responsabilité pour une formation médicale continue structurée et documentée, l'ISFM ne se contente pas du rapport prévu sur la formation continue. Il a aussi recommandé aux délégués de poursuivre le développement de la plate-forme de formation continue décidé initialement en 2015. Werner Bauer s'est montré convaincu: «Une plateforme optimisée renforce la crédibilité de la formation médicale continue. Elle permet au corps médical de garder la main et de rendre superflue toute intervention de l'État.» L'assemblée plénière a approuvé à l'unanimité la précision requise dans la Réglementation pour la formation continue, à savoir:

- L'obligation de formation continue englobe aussi l'obtention d'un diplôme de formation continue.

- Les sessions de formation continue devront être inscrites sur la plate-forme de formation continue de l'ISFM dès qu'elle aura fait peau neuve.

- Les sociétés de discipline contrôlent les sessions de formation continue au moins de façon ponctuelle. Jusqu'ici, beaucoup de sociétés de discipline octroient les diplômes de formation continue sur la seule base d'une autodéclaration, ce qui pénalise la crédibilité de la formation continue.

\section{Développement de la plate-forme de formation continue}

D'après le responsable de projet Johnny Graber, la plate-forme de formation continue permettra d'ici six ans de déclarer confortablement la formation continue au moyen d'un code $\mathrm{QR}$. Les sociétés de discipline et d'autres organisateurs pourront profiter d'une inscription et d'une reconnaissance efficace de leur manifestation, sans faille ni papier. Le développement est prévu en trois étapes de deux ans que l'assemblée plénière réévalue après chaque étape. Cette manière de procéder prudente et les avantages de la plate-forme numérique ont finalement aussi convaincu les voix sceptiques qui craignaient une surcharge administrative et financière. Les délégués ont donc approuvé la 


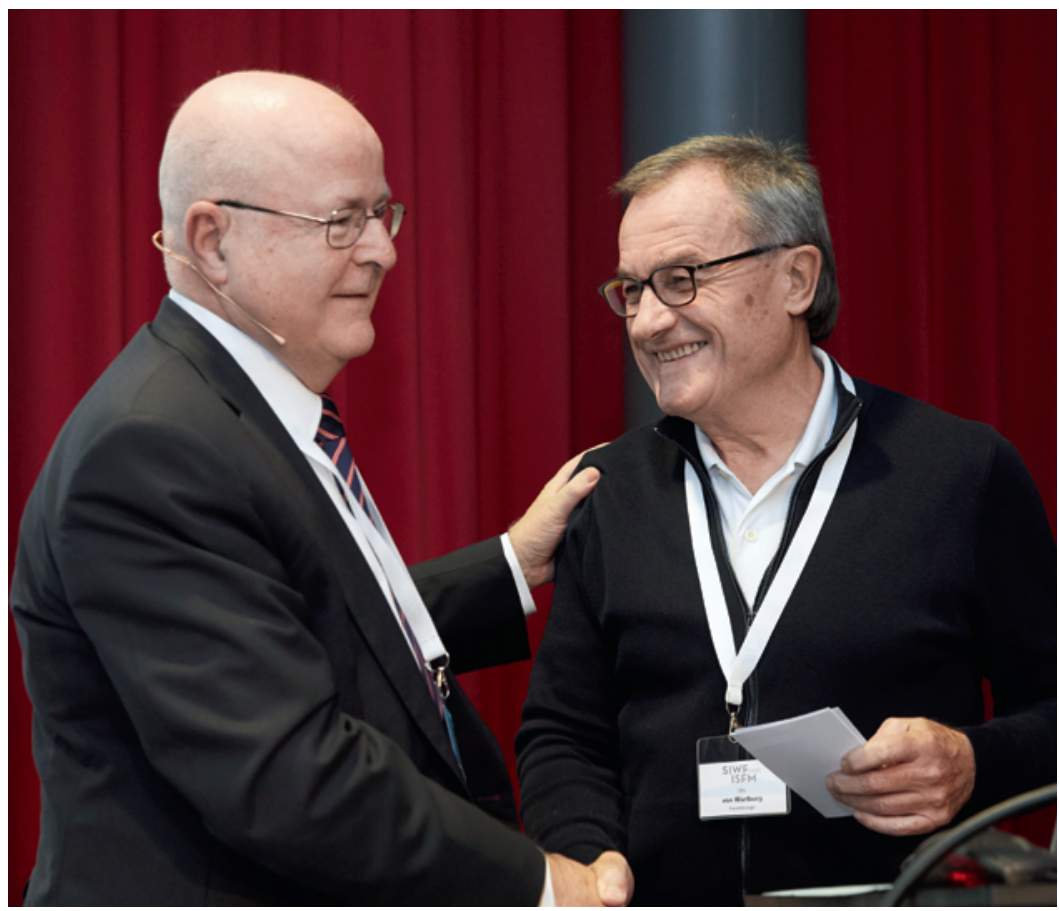

Urs von Wartburg remercie Werner Bauer pour 10 ans de succès à la tête de I'ISFM. (yk)

Christoph Hänggel ch[at]fmh.ch

\begin{abstract}
Assemblée plénière de l'ISFM
Organe autonome de la FMH, I'Institut suisse pour la formation médicale postgraduée et continue (ISFM) réunit tous les acteurs et organismes principaux du domaine de la formation médicale postgraduée et continue, et garantit aux médecins une formation de qualité élevée dans plus de 120 domaines spécialisés. L'assemblée plénière de I'ISFM remplit différentes fonctions. D'une part, les membres du plénum forment un organe doté d'un pouvoir décisionnel et électif, traitant les affaires relevant de son domaine de compétences à l'instar de la Chambre médicale de la FMH. De I'autre, l'assemblée, à laquelle sont également conviés des invités divers, constitue une plate-forme permettant de mettre à jour des informations importantes sur des thèmes spécifiques, d'échanger des expériences et de débattre de toutes sortes de sujets.
\end{abstract}

deuxième phase de développement par 39 oui, 4 non et 2 abstentions.

Le financement a par contre donné lieu à des discussions. Christoph Hänggeli, directeur, a affirmé que pour l'ISFM, il était évident que la formation continue, jusqu'ici déficitaire, ne pouvait plus faire l'objet d'un financement croisé par la formation postgraduée. Après une intervention marquée de l'ASMAC et de deux plus petites sociétés de discipline, l'assemblée plénière a finalement approuvé à l'unanimité l'augmentation de la taxe du diplôme de formation continue. Elle a opté pour un modèle proportionnel au lieu du montant fixe pour les sociétés de discipline.

\section{Remerciements à Werner Bauer}

L'assemblée plénière s'est terminée par un discours totalement imprévu à l'adresse du président sortant. Urs von Wartburg a chaleureusement remercié Werner Bauer pour son grand engagement tout au long de ces années, pour son sens intarissable de l'innovation et son énergie créatrice, son talent de négociateur et sa manière si intelligente d'avoir mis à profit son expérience au service de la formation médicale. Les délégués ont appuyé ces mots par un tonnerre d'applaudissements, certainement aussi en sachant que le travail d'anticipation fourni par Werner Bauer a permis de poser les conditions optimales à une formation médicale postgraduée et continue indépendante et tournée vers l'avenir.

\section{Références}

1 «Mégatendances et tournants»; compte rendu du symposium MedEd 2019 par Bruno Kesseli dans le Bulletin des médecins suisses 2019/49

2 www.siwf.ch $\rightarrow$ L'ISFM en bref $\rightarrow$ Prises de position $\rightarrow$ Lernzielformulierung und Assessments (en allemand)

3 www.siwf.ch $\rightarrow$ Établissements $\rightarrow$ Enquête auprès des médecinsassistants

4 www.siwf.ch $\rightarrow$ Projets $\rightarrow$ Entrustable Professional Activities (EPA)

Crédits photo

Fabienne Hohl (fh) et Yoshiko Kusano (yk) 